\title{
Empirical estimate of Ly $\alpha$ escape fraction in a statistical sample of Ly $\alpha$ emitters
}

\author{
H. Atek ${ }^{1}$, D. Kunth ${ }^{1}$, D. Schaerer ${ }^{2,3}$, M. Hayes ${ }^{2}$, J. M. Deharveng ${ }^{4}$, G. Östlin ${ }^{5}$, and J. M. Mas-Hesse ${ }^{6}$ \\ 1 Institut d'Astrophysique de Paris (IAP), 98bis boulevard Arago, 75014 Paris, France \\ e-mail: atek@iap.fr \\ 2 Observatoire de Genève, Université de Genève, 51 Ch. des Maillettes, 1290 Sauverny, Switzerland \\ 3 Laboratoire d'Astrophysique de Toulouse-Tarbes, Université de Toulouse, CNRS, 14 Avenue E. Belin, 31400 Toulouse, France \\ ${ }^{4}$ Laboratoire d'Astrophysique de Marseille, UMR 6110 CNRS/Université de Provence, 38 rue Joliot-Curie, \\ 13388 Marseille Cedex 13, France \\ 5 Oskar Klein Center for Cosmoparticle physics, Department of Astronomy, Stockholm University, 10691 Stockholm, Sweden \\ 6 Centro de Astrobiología (CSIC-INTA), POB 78, E28691 Villanueva de la Cañada, Madrid, Spain
}

Received 29 June 2009 / Accepted 8 September 2009

\section{ABSTRACT}

\begin{abstract}
Context. The Lyman-alpha ( $\operatorname{Ly} \alpha)$ recombination line is a fundamental tool for galaxy evolution studies and modern observational cosmology. However, subsequent interpretations are still prone to a number of uncertainties. Besides numerical efforts, empirical data are urgently needed for a better understanding of the Ly $\alpha$ escape process.

Aims. We empirically estimate the Ly $\alpha$ escape fraction in a statistically significant sample of galaxies in a redshift range $z \sim 0-0.3$. This estimate will constrain interpretations of current high-redshift Ly $\alpha$ observations.

Methods. An optical spectroscopic follow-up of a sub-sample of 24 Ly $\alpha$ emitters detected by GALEX at $z \sim 0.2-0.3$, combined with a UV-optical sample of local starbursts, both with matched apertures, allow us to quantify the dust extinction through Balmer lines, and to estimate the $\mathrm{Ly} \alpha$ escape fraction from the $\mathrm{H} \alpha$ flux corrected for extinction in the framework of the recombination theory.

Results. The global escape fraction of $\operatorname{Ly} \alpha$ radiation spans a wide range of values and $f_{\text {esc }}(\operatorname{Ly} \alpha)$ clearly decreases with increasing nebular dust extinction $E(B-V)$. Several objects show $f_{\text {esc }}(\mathrm{Ly} \alpha)$ greater than $f_{\text {esc }}$ (continuum), which may be taken as observational evidence for a clumpy ISM geometry or for an aspherical ISM. Selection biases and aperture size effects may still prevail between $z \sim 0.2-0.3$ Lyman-alpha emitters (LAEs) and local starbursts and may explain the difference observed for $f_{\text {esc }}(\operatorname{Ly} \alpha)$.
\end{abstract}

Key words. galaxies: starburst - galaxies: ISM - ultraviolet: galaxies - ISM: dust, extinction

\section{Introduction}

Considerable progress has been made in the last years in the detection and characterization of distant galaxy populations thanks, in particular, to 8-10 m class telescopes with large field of view instruments. In this context, the $\operatorname{Ly} \alpha$ emission line is of particular interest, since it remains the brightest spectral signature of remote young galaxies (Partridge \& Peebles 1967; Schaerer 2003). As a result, high redshift galaxies are now being routinely detected thanks to the Lyman Break selection and/or emission line surveys (e.g. Gronwall et al. 2007; Ouchi et al. 2008; Nilsson et al. 2009, and references therein); this situation is likely to improve with upcoming Extremely Large Telescopes (ELTs) and the James Webb Space Telescope (JWST). A comparable survey is now available for the first time at low redshift $(z \sim 0.2-0.35$, Deharveng et al. 2008) thanks to the GALEX (Galaxy Evolution Explorer) UV capabilities.

The Ly $\alpha$ line proves an invaluable tool in a cosmological context and is used in a wide variety of applications. Ly $\alpha$ has been used in recent studies to probe early stages of galaxy formation, estimate the star formation rate, trace large scale structures, identify potential hosts of population III stars and place constraints on cosmic reionization at $z \gtrsim 6$. However, in order to ensure a proper interpretation of these very promising Ly $\alpha$-oriented studies, one first must establish a robust calibration of the many parameters that control the complex transport of this line. The determination of the amount of Ly $\alpha$ radiation that escapes from the host galaxy is certainly the most important step toward understanding how various galaxy properties may distort the interpretation of Ly $\alpha$ observations. Although the order of importance of these parameters has been extensively discussed (e.g. Verhamme et al. 2008; Schaerer \& Verhamme 2008; Atek et al. 2009), empirical evidence based upon a large sample of galaxies is still missing.

From the International Ultraviolet Explorer (IUE) to the Hubble Space Telescope (HST) era, spectroscopic and imaging observations of nearby star forming galaxies have played a key role in identifying the main parameters responsible for the Ly $\alpha$ escape in a given galaxy. Recent high resolution Ly $\alpha$ imaging results clearly demonstrate the importance of resonant scattering, evidenced locally by very high $\mathrm{Ly} \alpha / \mathrm{H} \alpha$ ratios and an outstanding large Ly $\alpha$ scattering halo (e.g. Hayes et al. 2007; Atek et al. 2008; Östlin et al. 2009). However, most results so far have no statistical bearing and are still difficult to generalize, because the sample is not only small but consists of specific "hand-picked" objects. We propose here to improve this situation by using a larger sample of 24 Ly $\alpha$ emitting galaxies at $0.2 \lesssim z \lesssim 0.35$ found by GALEX. We have carried out a spectroscopic follow-up of a southern sub-sample with EFOSC2 on the ESO New Technology Telescope (NTT). This enables us to analyze how the Ly $\alpha$ emission is related to many physical properties of galaxies. We also re-analyzed UV-optical spectra of 11 local starbursts. For the first time, these large aperture 
Table 1. NTT EFOSC2 observations.

\begin{tabular}{llccc}
\hline \hline Mode & Slit & Exptime (s) & Nb. Obj. & Grism \\
\hline Spectroscopy & $1^{\prime \prime}$ & 3000 & 24 & 13 \\
Spectrophotometry & $5^{\prime \prime}$ & 1800 & 20 & 13 \\
\hline
\end{tabular}

observations allow us to determine empirically the Ly $\alpha$ escape fraction in a large sample of galaxies and to examine its dependence on dust extinction, if any.

\section{Observations}

\subsection{The GALEX sample}

$96 \operatorname{Ly} \alpha$ emitting galaxies at $z \sim 0.2-0.35$ were found by Deharveng et al. (2008) in the far ultraviolet (1350 $-1750 \AA$ ) from a GALEX slitless spectroscopic survey. Five fields covering a total area of $5.65 \mathrm{deg}^{2}$ were used to extract all continuum spectra with a minimum signal-to-noise ratio $(\mathrm{S} / \mathrm{N})$ per resolution element of 2 in the FUV. Ly $\alpha$ emitters are then visually selected on the basis of a potential Ly $\alpha$ emission feature, which naturally leads to a threshold of $E W_{\mathrm{Ly} \alpha} \gtrsim 10 \AA$. Data reduction and field characteristics are described in more detail in Deharveng et al. (2008).

\subsection{Spectroscopic follow-up}

Spectroscopic observations of 24 of the 31 galaxies in the Chandra Deep Field South (CDFS) and ELAIS-S1 fields were performed with EFOSC2 on the NTT at ESO La Silla. Observational conditions were very good with photometric sky and sub-arcsec seeing $\left(0.5^{\prime \prime}-1^{\prime \prime}\right)$. Two instrumental setups were used in long slit mode: (1) a spectrophotometric mode with a $5^{\prime \prime}$ slit, allowing observations to encompass the whole galaxy (20 out of 24 objects); and (2) a spectroscopic mode with a $1^{\prime \prime}$ slit, giving a better spectral resolution enabling us to correct $\mathrm{H} \alpha$ data for NII contamination. Both settings were used in combination with Grism \#13, covering a large wavelength range in the optical domain (3690-9320 ). A binning of $2 \times 2$ is used and corresponds to a plate-scale of $0.24^{\prime \prime} \mathrm{px}^{-1}$ and a spectral resolution of $F W H M \sim 12 \AA$ (for $1^{\prime \prime}$ slit spectra). To avoid second order contamination that affects the longer wavelength range, an order sorting filter has been mounted to cut off light blue-ward of $4200 \AA$. Observational settings and the mean exposure time per object are summarized in Table 1 .

The EFOSC2 spectra were reduced and calibrated using standard IRAF routines. The aperture extraction of 1D spectra was performed through the DOSLIT task. Spectra were flux calibrated using a mean sensitivity function determined by observations of standard stars (Feige110, HILT600, LTT1020, EG21) from the Oke (1990) catalog.

\subsection{IUE starburst sample}

We have re-analyzed UV-optical spectra of 11 local starburst galaxies, presented in McQuade et al. (1995) and StorchiBergmann et al. (1995) (see also Giavalisco et al. 1996) that are distant enough to separate the Ly $\alpha$ feature of the galaxy from geocoronal Ly $\alpha$ emission. In this way, we have complete control on the Ly $\alpha$ emission measurement method. Indeed, the definition of a Ly $\alpha$ emitter/absorber could be ambiguous for P Cygni profiles or an emission blended with absorption. Therefore, we consider here only the net Ly $\alpha$ flux. Data consist of IUE UV (1200-3300 ^) spectra combined with ground-based optical spectra with a matched aperture $\left(20^{\prime \prime} \times 10^{\prime \prime}\right)$. The line measurements have been performed following the same procedure used for the NTT spectra. We included in our analysis only net Ly $\alpha$ emitters, i.e. with $E W_{\mathrm{Ly} \alpha}>0$. Furthermore, for common objects between IUE and the Atek et al. (2008) samples, we retained the latter to minimize aperture size effects (see below). We then obtained five IUE objects.

\subsection{Emission line measurements}

All spectra were analyzed using the SPLOT package in IRAF. The redshift was measured using the wavelength position of several lines and line measurements were performed interactively on rest-frame spectra. We confirm with a better accuracy the redshift determinations based on the blind search for Ly $\alpha$ features in the GALEX survey.

Fluxes and equivalent widths (EWs) were measured for $\mathrm{H} \alpha$, $\mathrm{H} \beta$ and [NII] 6548, $6584 \AA$ A. For most spectra, the $\mathrm{H} \alpha$ line $(6563 \AA)$ is blended with [NII] lines, even for the $1^{\prime \prime}$ slit observations. In this case, a deblending routine is used within SPLOT to measure individual fluxes in each line. Then, the $\mathrm{NII} / \mathrm{H} \alpha$ line ratio is used to correct the spectrophotometric observations for [NII] contamination. It appears that the dust extinction (cf. Sect. 2.5) is sensitive to the aperture size, since our $1^{\prime \prime}$ slit observations, targeting the center of the galaxies, led in general to a higher extinction. But this does not affect the NII correction, which is relatively insensitive to dust extinction. To correct for underlying stellar absorption in the Balmer lines, we assumed a constant equivalent width of $2 \AA$, typical for starburst galaxies (Tresse et al. 1996; González Delgado et al. 1999) .

To determine uncertainties in the line fluxes, we ran 1000 Monte Carlo simulations in which random Gaussian noise, based on the data noise, is added to a noise-free spectrum. Then, emission lines were fitted. The computed MC errors depend essentially on the $\mathrm{S} / \mathrm{N}$ quality of spectra. Error propagation is applied through the calculation of all the quantities described above and the line ratios, extinction etc, computed hereafter.

Using BPT diagrams (Baldwin et al. 1981; Veilleux \& Osterbrock 1987), and Chandra X-ray observations, we have identified at most three galaxies possibly excited by an active galactic nucleus (AGN), which represents up to $12.5 \%$ of our sample. Studying similar $z \sim 0.3$ samples, Scarlata et al. (2009, submitted) find a comparable value (17\%), while Finkelstein et al. (2009a) claim a much higher fraction (around 43\%). We therefore removed these objects from our analysis. This diagnostic will be thoroughly addressed in Atek et al. (in prep.).

\subsection{Extinction}

Reddening along the galaxy line of sight is caused by interstellar dust extinction. The reddening contribution of our Galaxy is negligible for our objects. Then, the extinction coefficient, $C(\mathrm{H} \beta)$, intrinsic to the observed object can be calculated using the Balmer ratio between $\mathrm{H} \alpha$ and $\mathrm{H} \beta$ :

$\frac{f(\mathrm{H} \alpha)}{f(\mathrm{H} \beta)}=R \times 10^{-C[S(\mathrm{H} \alpha)-S(\mathrm{H} \beta)]}$

where $\mathrm{f}(\mathrm{H} \alpha)$ and $\mathrm{f}(\mathrm{H} \beta)$ are the measured integrated fluxes and $R$ is the intrinsic Balmer ratio. We use here a value of $R=2.86$, assuming case B recombination theory and a temperature of $10^{4} \mathrm{~K}$ (Osterbrock 1989). $S(\mathrm{H} \alpha)$ and $S(\mathrm{H} \beta)$ are determined from the Cardelli et al. (1989) extinction law. The colour excess $E_{B-V}$ is then simply computed using Eq. (1) and the relation 


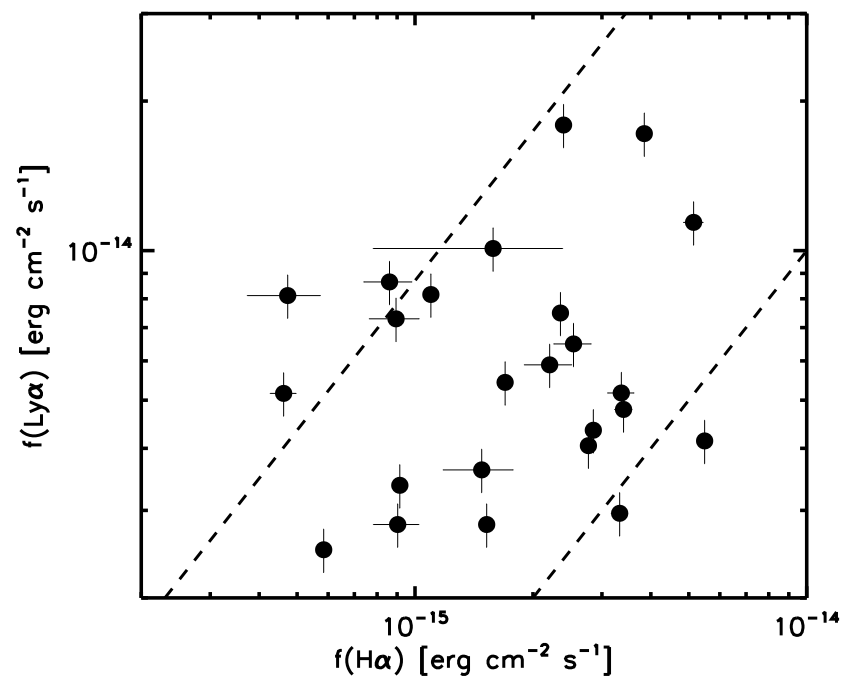

Fig. 1. Ly $\alpha$ versus $\mathrm{H} \alpha$ fluxes for the GALEX sample. The dashed line represent $\operatorname{Ly} \alpha / \mathrm{H} \alpha=8.7$ (case B) and $\mathrm{Ly} \alpha / \mathrm{H} \alpha=1$. The local objects (not represented here) show higher fluxes and lie in general close to the line 1:1.

$E(B-V)=C / 1.47$. Due to photometric calibration errors and probably a stronger stellar absorption than assumed here, few objects have a negative extinction with large uncertainties. This could also be the result of enhanced $\mathrm{H} \beta$ emission from a reflection nebula.

\section{Ly $\alpha$ escape fraction}

In Fig. 1, the $\operatorname{Ly} \alpha$ flux is plotted against the $\mathrm{H} \alpha$ one for the GALEX objects. While some objects show a Ly $\alpha / \mathrm{H} \alpha$ ratio consistent with the case B value, most of them lie well below this line. On the other hand, few objects show a $\operatorname{Ly} \alpha / \mathrm{H} \alpha>8.7$ at a significant level. The heavily attenuated values can be explained by the high extinction in the UV compared to the optical and to resonant scattering of $\operatorname{Ly} \alpha$ that increases the absorption optical depth. However, the observed dispersion and the points above the case B line could be the result of other parameters that will be discussed below. A convenient way to constrain the dust extinction parameter is to determine the escape fraction of $\operatorname{Ly} \alpha$ as a function of $E(B-V)$.

To determine the Ly $\alpha$ escape fraction we follow Atek et al. (2008). The method relies on the fact that $\mathrm{H} \alpha$ emission is not prone to complex radiation transport effects but is only affected by dust attenuation. Therefore, correcting the observed $\mathrm{H} \alpha$ flux for extinction while assuming a case B recombination theory (Osterbrock 1989), one can estimate the intrinsic Ly $\alpha$ flux. The Ly $\alpha$ escape fraction is then given by:

$f_{\mathrm{esc}}(\mathrm{Ly} \alpha)=f(\operatorname{Ly} \alpha) /\left(8.7 \times f(\mathrm{H} \alpha)_{\mathrm{C}}\right)$,

where $f(\operatorname{Ly} \alpha)$ is the observed flux and $f(\mathrm{H} \alpha)_{\mathrm{C}}$ is the extinctioncorrected $\mathrm{H} \alpha$ flux.

Figure 2 shows our empirical $f_{\text {esc }}$ values as a function of the nebular extinction for the GALEX and IUE samples described above. $f_{\text {esc }}$ values for 4 nearby galaxies from Atek et al. (2008), with $E W_{\mathrm{Ly} \alpha}>0 \AA$, are also shown. This figure summarizes much information with several implications for Ly $\alpha$ physics.

First, we find that $f_{\text {esc }}$ is not constant. It spans a wide range of values, typically from $f_{\text {esc }} \sim 0.5$ to $100 \%$ in the GALEX sample. Second, the Ly $\alpha$ escape fraction is clearly sensitive to the dust extinction and an anti-correlation is observed. We performed a

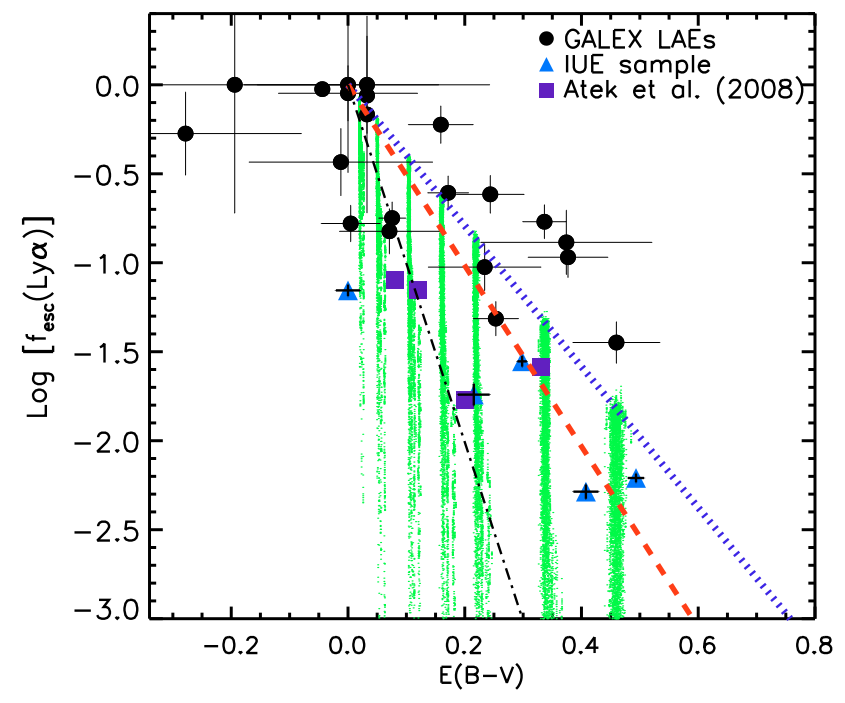

Fig. 2. Ly $\alpha$ escape fraction as a function of dust extinction, observed in $z \sim 0.3$ Ly $\alpha$ galaxies. The red dashed line represents the best fit to our entire sample (GALEX, IUE, and Atek et al. samples). The dark dotdashed line represent the best fit determined by Verhamme et al. (2008) from spectral fitting of $z \sim 3$ LBGs. The blue dotted line corresponds to the escape fraction of the continuum attenuated by dust extinction using the Cardelli et al. (1989) law. Green points are predictions for $f_{\text {esc }}(\mathrm{Ly} \alpha)$ using a 3D Ly $\alpha$ radiation transfer code (Verhamme et al. 2008). See text for details.

linear least-squares fit to this anti-correlation. The red dashed curve is the best solution found corresponding to

$f_{\text {esc }}(\operatorname{Ly} \alpha)=10^{-0.4 k(\operatorname{Ly} \alpha) E(B-V)} ; \quad k(\operatorname{Ly} \alpha) \sim 12.7 \pm 0.4$.

The extinction coefficient at $\operatorname{Ly} \alpha$ wavelength goes from $k(1216) \sim 9.9$ to 12.8 for Cardelli et al. (1989) and Calzetti et al. (2000) laws, respectively. Here, $k(\operatorname{Ly} \alpha)$ derived from the fit takes into account the averaged effects of all processes affecting the Ly $\alpha$ escape, such as the resonant scattering experienced by Ly $\alpha$ photons, which increases their mean path and therefore the effective dust optical depth, or velocity fields in the gas or the ISM geometry, which may ease the escape of Ly $\alpha$. The observed scatter around our mean relation is most likely indicative of this multi-parameter process. Using hydrodynamical cosmological simulations of Ly $\alpha$ emitters, Dayal et al. (2009) find similar trend between $f_{\mathrm{esc}}(\mathrm{Ly} \alpha)$ and extinction. For similar extinctions, the Ly $\alpha$ escape fraction tends to be higher on average in GALEX Ly $\alpha$ galaxies than in local objects. In the $z \sim 0$ objects, $f_{\text {esc }}$ never exceeds $\sim 10 \%$, whereas it covers a wide range of values reaching $100 \%$ at $z \sim 0.3$. This difference may be the result of different selection effects. The GALEX galaxies are selected from their Ly $\alpha$ emission amongst spectra taken from a blind search, whereas the local objects are from a sample of specific, optically-selected starburst galaxies.

Third, several objects show $f_{\mathrm{esc}}(\mathrm{Ly} \alpha)$ greater than the escape fraction expected for the continuum near Ly $\alpha$ (blue dotted line) as given by $f_{\text {esc }}$ (cont) $=10^{-0.4 k(1216) E(B-V)}$, where the most favourable value $k(1216) \sim 9.9$ is adopted from the Cardelli et al. law. Although $\mathrm{Ly} \alpha / \mathrm{H} \alpha$ ratios exceeding the theoretical value have already been found in local starbursts (Atek et al. 2008), they only occur locally, in spatially resolved objects, where this easily can be explained by a local Ly $\alpha$ "excess" due to scattering. Here, in some objects, it is the "global" Ly $\alpha$ escape fraction determined from the integrated spectra that is found to be higher than expected from the most favourable (i.e. flattest) attenuation law. These objects may be observational evidence for 
a multi-phase configuration of the ISM (Neufeld 1991; Hansen \& Peng Oh 2006; Finkelstein et al. 2009b), where dust is primarily distributed in cold neutral clouds with an ionized inter-cloud medium. By reflecting on the cloud surface, Ly $\alpha$ photons will be easily transmitted through the ionized medium. Alternatively, Scarlata et al. (2009, submitted) have advocated for a clumpy dust distribution scenario able to reproduce the observed Ly $\alpha$, $\mathrm{H} \alpha$ and $\mathrm{H} \beta$ intensities without the need for a two-phase model responsible for different paths for $\mathrm{Ly} \alpha$ and $\mathrm{H} \alpha$.

Approximately $2 / 6$ of these objects also show a relatively high Ly $\alpha$ equivalent width ( $E W_{\mathrm{Ly} \alpha} \sim 100-150 \AA$ ), as may be expected for a clumpy ISM. Furthermore, $f_{\text {esc }}(\operatorname{Ly} \alpha)$ higher than the UV continuum could also be due to orientation effects in objects with an aspherical ISM, e.g. in conical outflows, into which Ly $\alpha$ would be "channeled" more effectively than continuum radiation. The Ly $\alpha$ escape fractions of objects below the attenuation curve of the continuum can be quantitatively reproduced by models using a homogeneous ISM. However, this does not exclude that clumping may also play a role in these objects.

We performed an extensive grid of 3D Ly $\alpha$ radiation transfer simulations in homogeneous, spherically expanding shells (Hayes et al. in prep.), around a central emitting source of UV continuum plus the Ly $\alpha$ line, using an updated version of the MCLya code (Verhamme et al. 2006). We overplot in Fig. 2 the predictions of $f_{\text {esc }}$ as a function of $E(B-V)$ by using all possible values of the remaining parameters affecting $f_{\text {esc }}$ : the expansion velocity of the neutral gas, HI column density, Doppler parameter $b$ and $F W H M(\operatorname{Ly} \alpha)$ of the input emission line (for details, see Atek et al. 2009). The model $E(B-V)$ is computed from the predicted UV continuum attenuation, assuming the same extinction law as above. The model grid covers the bulk of the observed variations in $f_{\text {esc }}$ and $E(B-V)$. The homogeneous, spherical shell models may in principle be able to explain the majority of the objects, although tailored models including all observational constraints are needed to confirm this. However, the $f_{\text {esc }}$ values above the continuum attenuation curve (blue dotted line in Fig. 2) observed in 6 objects cannot be explained with these models, since Ly $\alpha$ photons cannot be less attenuated than the continuum in a homogeneous ISM. New radiation transfer computations in clumpy media are underway to examine this interesting behavior.

\section{Discussion}

We have presented here an estimation of the mean Ly $\alpha$ escape fraction as a function of the extinction and how different parameters can alter this simple relation. We have carefully chosen our aperture (5" slit) in optical spectroscopy in order to obtain Balmer fluxes comparable to Ly $\alpha$ ones obtained by the GALEX grism. This allows us to estimate the extinction and the escape fraction for the whole galaxy.

To keep the same consistency between the different samples, we decided to minimize selection effects by retaining only the net Ly $\alpha$ emitters in both the IUE and the imaging samples. We recall that the retained IUE large aperture $\left(20^{\prime \prime} \times 10^{\prime \prime}\right)$ UV observations and ground-based optical spectroscopy of local starbursts are all aperture matched. Furthermore, for Ly $\alpha$ imaging objects, the HST aperture is large enough to encompass the entire Ly $\alpha$ emission region. However, while the slitless mode of GALEX enables us to recover the diffuse Ly $\alpha$ emission, this is not necessarily the case for IUE observations. The size of the large aperture may remain insufficient for some nearby objects in order to encompass the scattered photons across a large area of the galaxy, as usually indicated by the large extent of the HI gas.
Therefore, $f_{\mathrm{esc}}(\operatorname{Ly} \alpha)$ could be slightly underestimated. On the contrary, as mentioned earlier, the $z \sim 0.2-0.3$ objects are selected on the grounds of their Ly $\alpha$ strength. This will likely favor the high Ly $\alpha$ escape fractions. One should then keep in mind that these opposite effects contribute to stretch the deviation from our best fit of this compilation of data points. Similar Ly $\alpha$ equivalent width criteria are commonly applied to select high- $z$ LAEs, implying most likely relatively high escape fractions and a less severe discrepancy between $\operatorname{Ly} \alpha$ and non-resonant radiation for this class of objects. A blind search of Ly $\alpha$ emitters would then find lower $f_{\mathrm{esc}}(\operatorname{Ly} \alpha)$ than the GALEX objects. This is already found in our current double blind survey in $\operatorname{Ly} \alpha$ and $\mathrm{H} \alpha$ at $z \sim 2.2$, where an average escape fraction of $\sim 4.5 \%$ is measured (Hayes et al. in prep.).

In contrast with other emission lines, the dust extinction is only one of several parameters governing $f_{\mathrm{esc}}(\operatorname{Ly} \alpha)$, and the extent of the dispersion around the fit is a good illustration. Given the importance of an accurate estimation of $f_{\mathrm{esc}}(\operatorname{Ly} \alpha)$, one needs to quantify the kinematical effects by measuring the cold ISM velocity in these objects; an additional step toward a precise calibration of $f_{\text {esc }}(\operatorname{Ly} \alpha)$. A more detailed investigation of the physical properties and SED modeling of our sample will be carried out in subsequent publications (Atek et al., in prep.).

Acknowledgements. We thank Daniela Calzetti, who kindly put her UV-optical spectra of the IUE sample at our disposal. The work of D.S. and M.H. is supported by the Swiss National Science Foundation. H.A. and D.K. are supported by the Centre National d'Études Spatiales (CNES). G.Ö. is Royal Swedish Academy of Sciences Research Fellow supported by a grant from the Knut and Alice Wallenberg Foundation. G.Ö. acknowledges support from the Swedish research council. J.M.M.H. is partially funded by Spanish MICINN grant AYA2007-67965. This work is based on observations made with ESO Telescopes at La Silla Observatories under programme ID 082.B-0392.

\section{References}

Atek, H., Kunth, D., Hayes, M., Östlin, G., \& Mas-Hesse, J. M. 2008, A\&A, 488,491

Atek, H., Schaerer, D., \& Kunth, D. 2009, A\&A, 502, 791

Baldwin, J. A., Phillips, M. M., \& Terlevich, R. 1981, PASP, 93, 5

Calzetti, D., Armus, L., Bohlin, R. C., et al. 2000, ApJ, 533, 682

Cardelli, J. A., Clayton, G. C., \& Mathis, J. S. 1989, ApJ, 345, 245

Dayal, P., Ferrara, A., \& Saro, A. 2009, ArXiv e-prints

Deharveng, J.-M., Small, T., Barlow, T. A., et al. 2008, ApJ, 680, 1072

Finkelstein, S. L., Cohen, S. H., Malhotra, S., Rhoads, J. E., \& Papovich, C. 2009a, ArXiv e-prints

Finkelstein, S. L., Rhoads, J. E., Malhotra, S., \& Grogin, N. 2009b, ApJ, 691, 465

Giavalisco, M., Koratkar, A., \& Calzetti, D. 1996, ApJ, 466, 831

González Delgado, R. M., Leitherer, C., \& Heckman, T. M. 1999, ApJS, 125, 489

Gronwall, C., Ciardullo, R., Hickey, T., et al. 2007, ApJ, 667, 79

Hansen, M., \& Peng Oh, S. 2006, New Astron. Rev., 50, 58

Hayes, M., Östlin, G., Atek, H., et al. 2007, MNRAS, 382, 1465

McQuade, K., Calzetti, D., \& Kinney, A. L. 1995, ApJS, 97, 331

Neufeld, D. A. 1991, ApJ, 370, L85

Nilsson, K. K., Tapken, C., Moeller, P., et al. 2009, A\&A, 498, 13

Oke, J. B. 1990, AJ, 99, 1621

Osterbrock, D. E. 1989, Astrophysics of gaseous nebulae and active galactic nuclei, Research supported by the University of California, John Simon Guggenheim Memorial Foundation, University of Minnesota, et al. (Mill Valley, CA: University Science Books), 422

Östlin, G., Hayes, M., Kunth, D., et al. 2009, AJ, 138, 923

Ouchi, M., Shimasaku, K., Akiyama, M., et al. 2008, ApJS, 176, 301

Partridge, R. B., \& Peebles, P. J. E. 1967, ApJ, 147, 868

Schaerer, D. 2003, A\&A, 397, 527

Schaerer, D., \& Verhamme, A. 2008, A\&A, 480, 369

Storchi-Bergmann, T., Kinney, A. L., \& Challis, P. 1995, ApJS, 98, 103

Tresse, L., Rola, C., Hammer, F., et al. 1996, MNRAS, 281, 847

Veilleux, S., \& Osterbrock, D. E. 1987, ApJS, 63, 295

Verhamme, A., Schaerer, D., \& Maselli, A. 2006, A\&A, 460, 397

Verhamme, A., Schaerer, D., Atek, H., \& Tapken, C. 2008, A\&A, 491, 89 